\title{
Prevention of autoimmune diabetes by immunogene therapy using recombinant vaccinia virus expressing glutamic acid decarboxylase
}

\author{
H.-S. Jun ${ }^{1}$, Y.-H. Chung11, J. Han ${ }^{1}$, A. Kim ${ }^{1}$, S. S. Yoo ${ }^{1}$, R. S. Sherwin ${ }^{2}$, J.-W. Yoon ${ }^{1,2}$ \\ ${ }^{1}$ Laboratory of Viral Immunopathogenesis of Diabetes, Julia McFarlane Diabetes Research Centre, Faculty of Medicine, \\ The University of Calgary, Calgary, Alberta, Canada \\ ${ }^{2}$ Diabetes Endocrinology Research Center, School of Medicine, Yale University, New Haven, Connecticut, USA
}

\begin{abstract}
Aims/hypotheses. Type I (insulin-dependent) diabetes mellitus results from T-cell-mediated autoimmune destruction of pancreatic beta cells. Among the beta-cell autoantigens that have been implicated in triggering of beta-cell-specific autoimmunity, glutamic acid decarboxylase (GAD) is a strong candidate in both humans and the NOD mouse. We aimed to determine whether treatment with a recombinant vaccinia virus expressing GAD (rVV-GAD65) could prevent the development of diabetes in NOD mice.

Methods. Three-eight-to-nine-week-old female NOD mice were injected with various doses of rVV-GAD65 or rVV-MJ601as a control. We then examined the incidence of diabetes, T-cell proliferative response to GAD, amounts of anti-GAD IgGs, cytokine production and generation of regulatory cell populations.

Results. Administration of rVV-GAD65 to NOD mice prevented diabetes in an age-dependent and dose-dependent manner. Splenic T cells from rVV-GAD65treated mice did not proliferate in response to GAD65.
\end{abstract}

The amount of IgG1 was increased, whereas $\operatorname{IgG} 2 \mathrm{a}$ amounts did not change in rVV-GAD65-treated NOD mice. The production of interleukin-4 increased, whereas the production of interferon- $\gamma$ decreased in rVV-GAD65-treated mice after stimulation with GAD. Furthermore, splenocytes from rVV-GAD65treated NOD mice prevented the transfer of diabetes by splenocytes from acutely diabetic NOD mice in NOD.scid recipients.

Conclusion/interpretation. Immunogene therapy using a recombinant vaccinia virus expressing GAD results in the prevention of autoimmune diabetes in NOD mice by the induction of immunological tolerance through active suppression of effector $\mathrm{T}$ cells, and this treatment might have therapeutic value for the prevention of Type I diabetes. [Diabetologia (2002) 45:668-676]

Keywords Type I diabetes, NOD mice, recombinant vaccinia virus, glutamic acid, decarboxylase immunogene therapy, immunological tolerance, regulatory cells, effector T cells, Th2 immune response cytokine.
Received: 5 November 2001 and in revised form: 16 January 2002

Published online: 4 April 2002

C) Springer-Verlag 2002

Coresponding author: J.-W. Yoon, Laboratory of Viral Immunopathogenesis of Diabetes, Julia McFarlane Diabetes Research Centre, Faculty of Medicine, The University of Calgary, Calgary, Alberta, Canada, e-mail: yoon@ucalgary.ca

Abbreviations: GAD, Glutamic acid decarboxylase; IDDM, insulin dependent diabetes mellitus; NOD, non-obese diabetic; NS1, non-structural protein 1; PFU, plaque-forming units; rVV, recombinant vaccinia virus; scid, severe combined immunodeficiency
Type I (insulin-dependent) diabetes mellitus, results from the destruction of pancreatic beta cells by T cellmediated, beta-cell-specific autoimmune responses [1, $2,3,4,5,6]$. The non-obese diabetic (NOD) mouse is one of the best animal models for human Type I diabetes; it spontaneously develops the disease with many features in common with human Type I diabetes $[7,8,9]$. It has been shown that the destruction of pancreatic beta cells is mediated by both $\mathrm{CD}^{+}$and $\mathrm{CD}^{+}$ $\mathrm{T}$ cells $[10,11,12]$. Therefore, various strategies, particularly autoantigen-based strategies, have been used to tolerise these autoreactive $\mathrm{T}$ cells and prevent autoimmune Type I diabetes in NOD mice. In NOD mice 
and human Type I diabetes, glutamic acid decarboxylase (GAD) and insulin are known to be major betacell autoantigens that play an important role in the development of diabetes $[13,14]$.

To explore the potential of immunogene therapy based on GAD for the prevention of autoimmune diabetes, we developed a recombinant vaccinia virus (rVV) expressing GAD. Vaccinia virus has been used as a live vaccine against smallpox $[15,16]$. Of particular importance are the findings that $\mathrm{rVV}$ can induce humoral and cell-mediated immune responses to a target protein and that the induced immune responses are long lived $[17,18,19,20]$. For example, animals immunised with rVV expressing a protein from a pathogenic virus are protected from the disease caused by challenge with that virus $[21,22]$. Of note too, in Phase I clinical trials with vaccinia virus expressing the human immunodeficiency virus 1 envelope gene, no serious side effects were found in healthy subjects that received percutaneous administration [23, 24]. These advantages of rVV would make it valuable for clinical use in Type I diabetes, if the administration of rVV expressing beta-cell autoantigen proteins such as GAD can tolerise the autoreactive T cells and prevent the development of diabetes. In this study, we attempted to prevent autoimmune Type I diabetes in NOD mice using rVV expressing GAD65 protein (rVV-GAD65).

\section{Materials and methods}

Animals. NOD mice were obtained from Taconic (Germantown, N.Y., USA) and NOD.severe combined deficiency (scid) mice were obtained from Jackson Laboratory (Bar Harbor, Me., USA). Mice were bred and housed under specific pathogen-free conditions and provided with sterile food and water at the Animal Resources Centre of the University of Calgary. Female NOD mice were used throughout the experiments. The use and care of the animals in this study were approved by the Animal Care Committee of the University of Calgary.

Construction of recombinant vaccinia virus expressing mouse GAD65 protein. The mouse cDNA coding for GAD65 [25] was cloned into the vaccinia virus vector pMJ601 [26] under the strong synthetic late promoter using the NheI and BamHI sites (pMJ-GAD65). The rVV was produced by homologous recombination, as described elsewhere $[17,18]$. The recombinant plasmid $(10 \mu \mathrm{g})$ was transfected into CV-1 cells (ATCC, Manassas, Va., USA), which had previously been infected with wild type vaccinia virus $(0.05$ plaque forming units [PFU]/cell), using the calcium phosphate method. As a control, the plasmid pMJ601 was transfected into CV-1 cells, instead of pMJ-GAD65, to produce rVV-MJ601. The rVV was plaque purified on $\mathrm{HuTK}^{-143 B}$ cells (ATCC) in the presence of 5bromodeoxyuridine in agar overlay three times and amplified.

Immunisation with recombinant vaccinia viruses. Three-weekold female NOD mice were injected with $1 \times 10^{7}, 2.5 \times 10^{7}$ or $5 \times 10^{7}$ PFU i.p. of rVV-GAD65. As controls, age-matched and sex-matched NOD mice were injected with $1 \times 10^{7}, 2.5 \times 10^{7}$ or $5 \times 10^{7}$ PFU of rVV-MJ601 or rVV expressing an unrelated protein, the non-structural protein (NS)-1 of the Kilham rat virus (rVV-NS1) [27]. To determine whether the effect of rVV-GAD65 is age-dependent, 8-9 week-old female NOD mice also were injected with $5 \times 10^{7} \mathrm{PFU}$ i.p. of rVV-GAD65 or rVV-MJ601. The development of diabetes was monitored by the measurement of urine glucose using Diastix (Miles, Ont., Canada) and confirmed by measuring blood glucose using a one-touch Basic glucometer (Lifescan, Milpitas, Calif., USA). Any mouse with a blood glucose concentration of more than $16.7 \mathrm{mmol} / \mathrm{l}$ on three consecutive days was scored as diabetic.

In vitro proliferation assay. Single cell splenocytes were prepared from NOD mice 8 weeks after injection of $5 \times 10^{7}$ rVV-GAD65 or $5 \times 10^{7}$ rVV-MJ601 and from age-matched and sex-matched uninjected NOD mice. The cells $\left(5 \times 10^{5}\right)$ were cultured in $200 \mu \mathrm{l}$ of complete RPMI-1640 medium supplemented with $10 \%$ fetal bovine serum, $1 \mathrm{mmol} / \mathrm{l} \mathrm{L}$-glutamine, $100 \mathrm{U} / \mathrm{ml}$ penicillin, $100 \mu \mathrm{g} / \mathrm{ml}$ streptomycin and $50 \mu \mathrm{mol} / \mathrm{l}$ beta-mercaptoethanol in a 96-well microplate in the presence of recombinant human GAD65 protein (produced in baculovirus) or ovalbumin for $72 \mathrm{~h}$. The cells were then pulsed with ${ }^{3} \mathrm{H}$-thymidine $(1 \mu \mathrm{Ci} /$ well $)$ for $16-18 \mathrm{~h}$. The proliferation was quantified by determining the ${ }^{3} \mathrm{H}$-thymidine incorporation using a liquid scintillation counter.

Assay of antibodies against GAD. Sera from NOD mice were collected 8 weeks after injection with $5 \times 10^{7}$ rVV-GAD65 or $5 \times 10^{7}$ rVV-MJ601 and reacted with recombinant human GAD65 protein (produced in baculovirus) as described elsewhere $[28,29]$. GAD65 protein was coated onto a 96-well microplate $\left(10 \mu \mathrm{g} / \mathrm{ml}\right.$ in $\left.0.1 \mathrm{~mol} / \mathrm{NaHCO}_{3}, \mathrm{pH} 8.5\right)$ at $4^{\circ} \mathrm{C}$ overnight. Sera from age-matched and sex-matched NOD mice were used as a control. After washing, the bound antibody was reacted with alkaline phosphatase-conjugated goat anti-mouse $\mathrm{IgG}$, anti-mouse IgG1 or anti-mouse IgG2a antibodies. The colour was developed by incubation with 4-nitrophenyl phosphate. The optical density was read at $405 \mathrm{~nm}$ with an ELISA reader.

RT-PCR analysis. Total RNA was isolated from splenocytes of $5 \times 10^{7}$ rVV-GAD65- and $5 \times 10^{7}$ rVV-MJ601-immunized mice at 1, 2, 3 and 4 weeks after immunisation using a RNA extraction kit (Qiagen, Mississauga, Ont., Canada) according to the manufacturer's protocol. RNA from the splenocytes of agematched and sex-matched NOD mice was used as a control. Two $\mu \mathrm{g}$ of total RNA was converted to cDNA using Superscript II reverse transcriptase (Gibco BRL, Gaithersburg, Md., USA) and oligo (dT) $)_{12-18}$. PCR was carried out using specific primers for various cytokine genes, as described previously [30]. The primers used were as follows: IL-2: sense CTTGCCCAAGCAGGCCACAG, antisense - GAGCCTTATGTGTTGTAAGC; IFN- $\gamma$. sense - AGCTCTGAGACAATGAACGC, antisense - GGACAATCTCTTCCCCACCC; IL-4: sense - TCTTTCTCGAATCTACCAGG, antisense - CATGGTGGCTCAGTACTACG; and $I L-10$ : sense - CAAACAAAGGACCAGCTGGAC, antisense - TTGACCTCAGCGCTGAGTTG. Hypoxanthine phosphoribosyl transferase (HPRT) mRNA was amplified as an internal standard; the primers used for HPRT were: sense - GTAATGATCAGTCAACGGGGGAC, antisense - CAAGCAAGCTTGCAACCTTAACCA. The PCR conditions were optimised for each set of primers. PCR was done using different numbers of cycles to ensure that amplification occurred in a linear range. The PCR mixture $(50 \mu \mathrm{l})$ contained $0.2 \mathrm{mmol} / \mathrm{l}$ of deoxynucleotide triphosphate, $1 \mu \mathrm{mol} / \mathrm{l}$ of each specific primer, $1.5 \mathrm{mmol} / \mathrm{l}$ or $2 \mathrm{mmol} / \mathrm{l}$ $\mathrm{MgCl}_{2}, 50 \mathrm{mmol} / \mathrm{l} \mathrm{KCl}, 10 \mathrm{mmol} / \mathrm{l}$ Tris-Cl (pH 9.0) and 2.5 units of Taq polymerase (Pharmacia Biotech, Uppsala, Swe- 

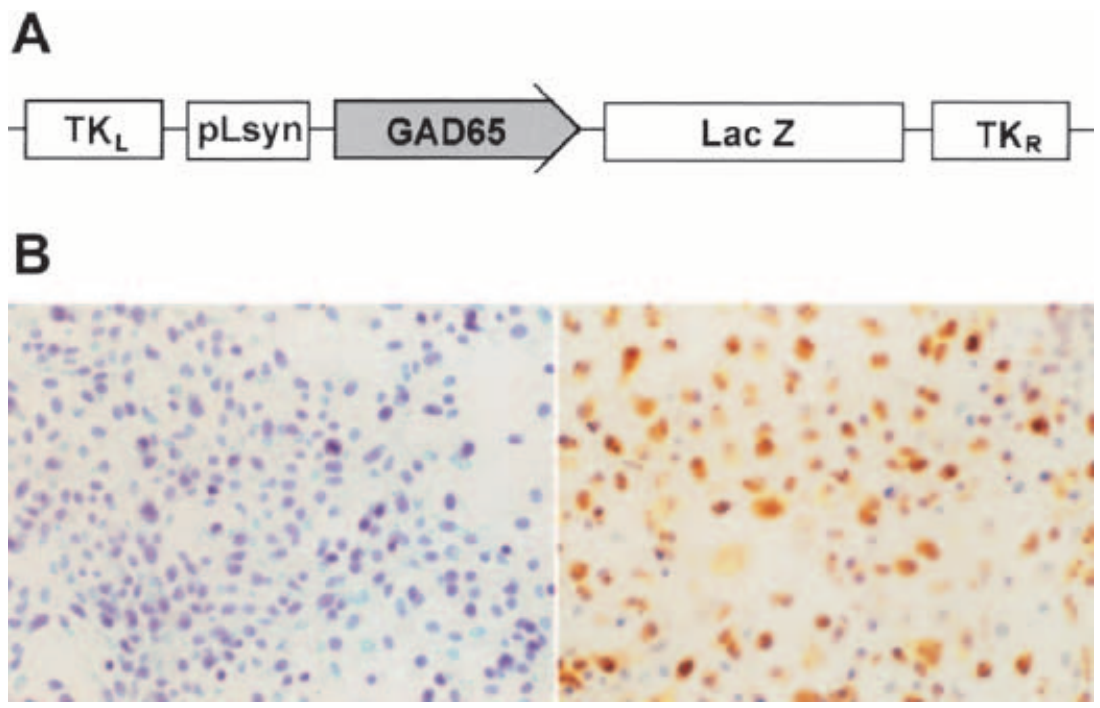

Fig. 1A, B. A Schematic structure of GAD-expressing vaccinia virus vector. Mouse GAD65 cDNA was inserted into the multiple cloning site of the vector, pMJ601, using BamHI and NheI restriction enzyme sites under the late synthetic promoter of vaccinia virus. $T K_{L}$, thymidine kinase left region; $T K_{R}$, thymidine kinase right region; $L a c Z$, beta-galactosidase; GAD65, mouse GAD65 cDNA. B Immunohistochemical staining of GAD65 expression in recombinant vaccinia virus. HeLa cells were infected with rVV-GAD65 or rVV-MJ601; the GAD65 protein was stained using an antibody to GAD, GAD1, and counterstained with haematoxylin and eosin. Left panel: HeLa cells infected with rVV-MJ601: Right panel: HeLa cells infected with $\mathrm{rVV}-\mathrm{GAD} 65$. Brown precipitate indicates the presence of immunoreactive GAD

den). After amplification, the products were subjected to electrophoresis on a $1.5 \%$ agarose gel and detected by ethidium bromide staining.

ELISA of cytokine production. The production of cytokines in the splenic T cells of rVV-injected NOD mice was measured as described previously [30]. Splenocytes were isolated 8 weeks after injection of $5 \times 10^{7}$ rVV-GAD65 or $5 \times 10^{7}$ rVV-MJ601. The cells were cultured in 24 -well plates $\left(2 \times 10^{6}\right.$ cells/well $)$ in complete RPMI-1640 medium in the presence or absence of GAD protein for $72 \mathrm{~h}$. The supernatant was collected and IFN- $\gamma$ and IL-4 production was measured by sandwich ELISA using a Quantikine kit (R \& D Systems, Minneapolis, Minn., USA).

Adoptive transfer of diabetes. Three week-old female NOD mice were injected with rVV-GAD65 or rVV-MJ601 $\left(5 \times 10^{7} \mathrm{PFU} / \mathrm{mouse}\right)$. At 20 weeks of age, splenocytes $\left(1 \times 10^{7}\right.$ cells) were isolated from each donor and injected i.p. with or without splenocytes $\left(1 \times 10^{7}\right.$ cells $)$ from uninjected, acutely diabetic NOD mice into 6 to 8 week-old NOD.scid mice. The development of diabetes was determined by the measurement of urine and blood glucose every other day. As controls, agematched NOD.scid mice received splenocytes $\left(1 \times 10^{7}\right.$ cells $)$ from uninjected, acutely diabetic NOD mice.

Immunohistochemistry. The expression of GAD65 protein was examined in rVV-GAD65-infected cells by immunohistochemistry using GAD1 monoclonal antibody (ATCC). Briefly, HeLa cells were cultured in Lab-Tek slide flasks (Nunc, Naperville,
Ill., USA) and infected with rVV-GAD65 or rVV-MJ601. At 3 days after infection, the cells were washed and fixed with acetone, incubated with GAD1 monoclonal antibody followed by horseradish peroxidase-conjugated secondary antibody, visualized using 3-3' -diaminobenzidine as a substrate (Vector Laboratories, Burlingame, Calif., USA) and counterstained with haematoxylin and eosin.

Histology. Mice that had been immunised with $5 \times 10^{7} \mathrm{rVV}$ GAD65 or $5 \times 10^{7}$ rVV-MJ601 at 3 weeks of age were killed at 25 and 40 weeks of age ( 5 mice/group) and the pancreata and salivary glands were removed. Each pancreas was fixed with $10 \%$ buffered formalin, embedded in paraffin, sectioned at $4.5 \mu \mathrm{m}$ and stained with haematoxylin and eosin [31]. Insulitis grade was determined as follows: 0 , normal islet; 1 , mononuclear infiltration, largely in the periphery, in less than $25 \%$ of the islet; $2,25 \%-50 \%$ of the islet showing mononuclear infiltration; 3 , over $50 \%$ of the islet showing mononuclear infiltration; 4, small, retracted islet with few mononuclear cells.

Statistical analyses. Statistical analyses were performed using Student's $t$ test. A $p$ value of 0.05 or less was considered to be statistically significant.

\section{Results}

Prevention of autoimmune diabetes and insulitis, but not sialitis, in NOD mice by immunisation with recombinant vaccinia virus expressing GAD. We cloned mouse GAD65 cDNA [25] into the vaccinia virus vector pMJ601 under the late synthetic promoter [26] (Fig. 1A) and produced rVV-GAD65. The expression of GAD protein was confirmed by immunohistochemistry; HeLa cells that had been infected with rVVGAD65 expressed GAD65 protein (Fig. 1B, right panel), while those infected with rVV-MJ601 (control rVV without GAD65 cDNA) did not (Fig. 1B, left panel). To determine whether $\mathrm{rVV}-G A D 65$ prevented the development of autoimmune diabetes in NOD mice, we injected rVV-GAD65 $\left(5 \times 10^{7}\right.$ PFU) i.p. into 3 week-old female NOD mice and examined the development of diabetes. Only 7\% (2/29) of the NOD 


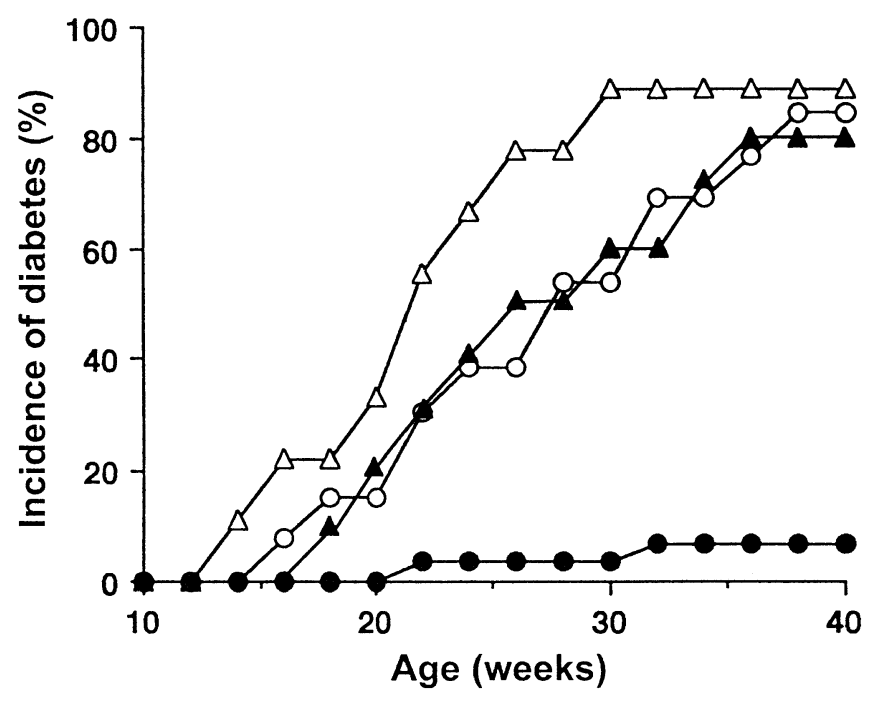

Fig. 2. Treatment of NOD mice with rVV-GAD65 at a young age prevented the development of diabetes. Three week-old female NOD mice were injected with rVV-GAD65 $\left(--_{-}, n=29\right)$, rVV-MJ601 $(-\bigcirc-, n=13)$ or rVV-NS1 $(-\mathbf{\Delta}-, n=10)\left(5 \times 10^{7}\right.$ PFU) and the development of diabetes was monitored until 40 weeks of age by observing the onset of hyperglycaemia. Uninjected mice $(-\triangle-, n=9)$ were used as a control

mice that received rVV-GAD65 developed diabetes, whereas $85 \%(11 / 13)$ and $80 \%(8 / 10)$ of the NOD mice that received $\mathrm{rVV}-\mathrm{MJ} 601$ or $\mathrm{rVV}-\mathrm{NS} 1$, respectively, developed diabetes by 40 weeks of age. Similarly, 89\% (8/9) of the uninjected control NOD female mice also developed diabetes by the same age (Fig. 2). These data suggest that the prevention of diabetes by rVV-GAD65 is GAD antigen-specific, and not simply a non-specific effect of the vaccinia virus infection itself. However, the onset of diabetes in the rVVMJ601- or rVV-NS1-infected mice was somewhat delayed compared with that in uninjected control NOD mice. The mean age of diabetes onset was 26 weeks in both the rVV-MJ601- and rVV-NS1-infected mice as compared to 21 weeks in the uninjected animals.

To determine whether the slight delay in the onset of diabetes in rVV-MJ601-injected or rVV-NS1-injected NOD mice could be attributed to a non-specific immune disturbance caused by rVV infection, we examined the cytokine gene expression of splenocytes at different times (1-4 weeks) after infection with the control virus. The expression of interleukin $(I L)$-2, interferon (IFN)- $\gamma, I L-4$ and $I L-10$ increased substantially 1 week after rVV-MJ601 or rVV-NS1 infection compared to uninfected control mice, thereafter cytokine expression gradually declined, reaching normal amounts 3 weeks after infection, similar to the amounts seen in uninfected mice (data not shown). This result suggests that the modest delay in disease onset in rVV-MJ601- or rVVNS1-injected NOD mice could be due to a non-specific, transient immune disturbance.

The development of insulitis in NOD mice injected with rVV-GAD65 was reduced compared to those in-

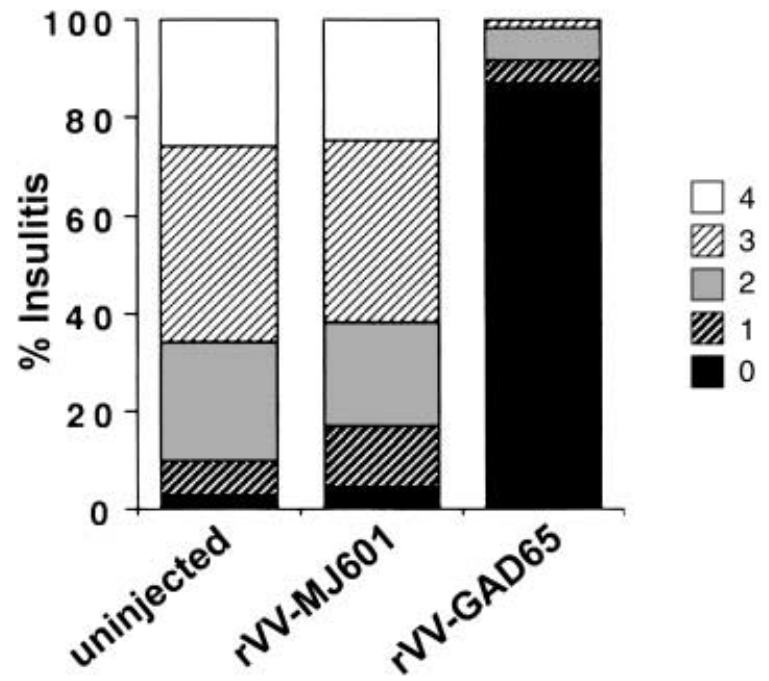

Fig. 3. Treatment of NOD mice with rVV-GAD65 prevented the development of insulitis. Three-week-old female NOD mice were injected with rVV-GAD65 or rVV-MJ601 $\left(5 \times 10^{7}\right.$ PFU). Non-diabetic animals $(n=5)$ were killed at 25 weeks of age and histological examination of pancreatic islets (at least 20 islets for each mouse) was performed. The insulitis score was determined as described in Methods

jected with rVV-MJ601. A total of $87 \%$ of the examined islets from $\mathrm{rVV}-\mathrm{GAD} 65$-injected NOD mice were intact (Figs. 3 and 4A), whereas $83 \%$ of the islets from rVV-MJ601-injected NOD mice showed moderate to severe insulitis, similar to that found in uninjected control NOD mice, at 25 weeks of age (Figs. 3 and 4B, $4 C)$. At 40 weeks of age, when the experiment was terminated, $42 \%$ of the examined islets from rVVGAD65-injected mice remained intact, $50 \%$ of the islets showed mild peri-insulitis or moderate insulitis, and only about $8 \%$ of the islets showed severe insulitis.

To determine whether the immunisation of NOD mice with rVV-GAD65 specifically affects beta-cellspecific autoimmunity, we examined the salivary glands, which show lymphocytic infiltration in NOD mice. There was equally severe infiltration of lymphocytes in the salivary glands of both rVV-GAD65- and rVV-MJ601-infected NOD mice at 25 weeks of age (Fig. 4D, E), similar to that of uninjected NOD mice (Fig. 4F). These results indicate that the suppression of autoimmunity induced by immunisation with rVVGAD65 encompasses only the pancreatic islets, and not other target tissues that do not express GAD protein.

Preventive effect of $r V V$-GAD65 is dependent upon both dosage and age of immunised NOD mice. The influence of rVV-GAD65 dosage on disease expression was evaluated by immunising three-week-old NOD female mice with three different dosages of rVV-GAD65 $\left(1 \times 10^{7}, 2.5 \times 10^{7}\right.$ and $5 \times 10^{7} \mathrm{PFU} / \mathrm{mouse}$, i.p.). Diabetes occurred in $67 \%(10 / 15)$ of the low dose $\left(1 \times 10^{7} \mathrm{PFU}\right)$ and $53 \%(8 / 15)$ of the intermediate dose $\left(2.5 \times 10^{7} \mathrm{PFU}\right)$ groups, as compared to $7 \%(2 / 29)$ in the high dose 

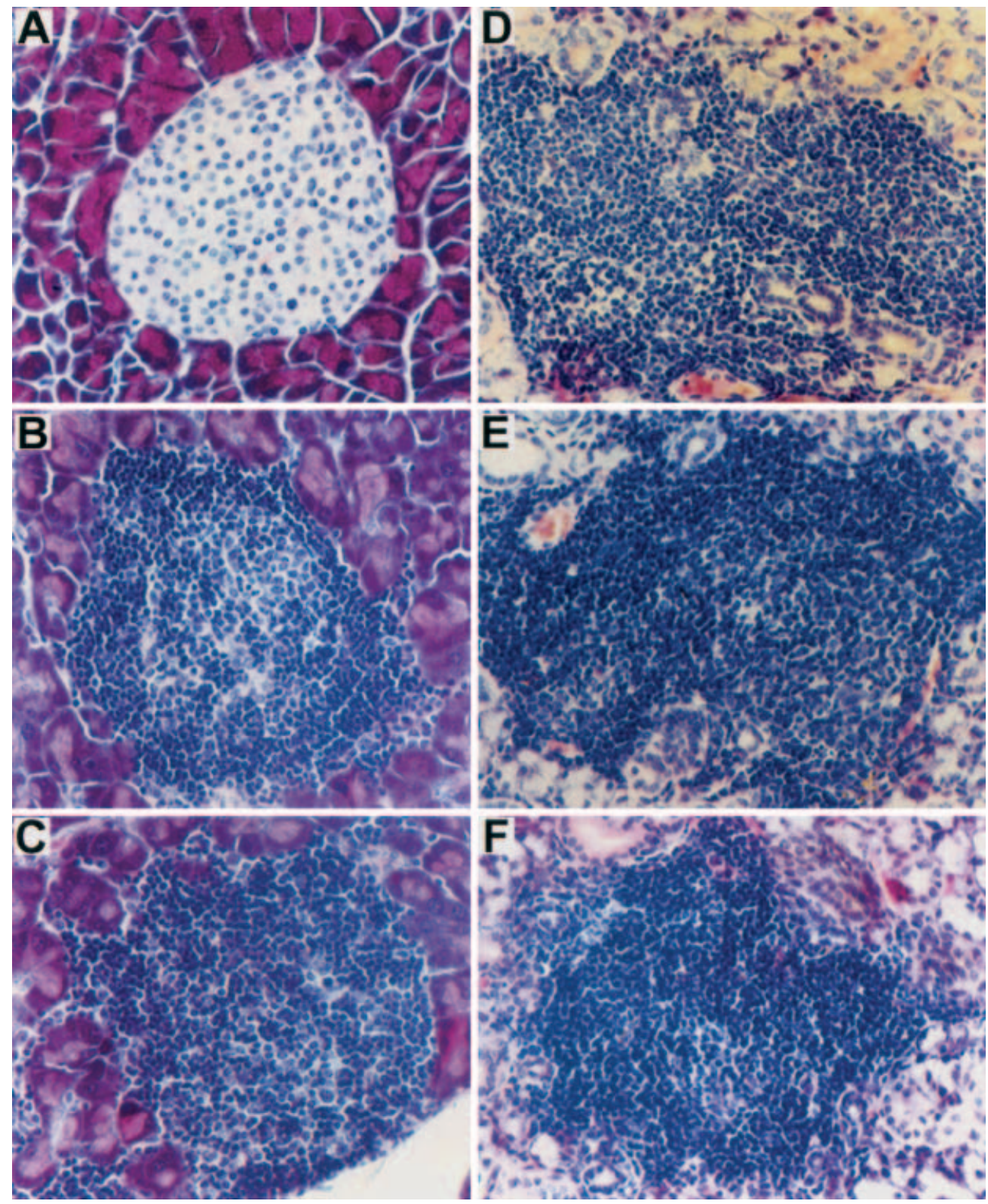

Fig. 4A-F. Photomicrographs of representative islets from: A rVV-GAD65-injected mice, B rVV-MJ601-injected mice and $\mathbf{C}$ uninjected mice, and salivary glands from: $\mathbf{D} \mathrm{rVV}$ GAD65-injected mice, $\mathbf{E}$ rVV-MJ601-injected mice and $\mathbf{F}$ uninjected mice at 25 weeks of age. Sections were stained with haematoxylin and eosin

$\left(5 \times 10^{7}\right.$ PFU) $\quad$ rVV-GAD65-immunised NOD mice. In addition, the time of rVV-GAD65 immunisation altered its effectiveness. When older NOD mice $(8$ to 9 weeks of age) were immunised with rVV-GAD65 (5×107 PFU/mouse), 73\% (8/11) became diabetic, values comparable to those of the unimmunised control NOD mice $(75 \%, 9 / 12)$. Thus, the dose of GAD-expressing recombinant vaccinia virus and the age of the NOD mice at the time of immunisation determine the effect of the vaccine on the prevention of diabetes.
rVV-GAD65 immunisation of NOD mice tolerises $T$ cell immune response to GAD and induces Th2 immune response. To determine the mechanism by which rVV-GAD65 prevented the development of diabetes, we first examined the proliferative response to GAD protein of splenocytes collected 8 weeks after injection with rVV-GAD65. These splenocytes did not respond to GAD (Fig. 5). In contrast, GAD stimulated the proliferation of splenocytes collected from rVV-MJ601-injected mice and uninjected control NOD mice (Fig. 5). Splenocytes from all three groups showed no proliferative response against ovalbumin, an antigen unrelated to beta-cell proteins. These results imply that GAD-reactive $\mathrm{T}$ cells are tolerated in rVV-GAD65-injected animals. We also examined the humoral immune response against GAD in the sera of NOD mice 8 weeks after injection of rVV-GAD65 or rVV-MJ601. There was an in- 


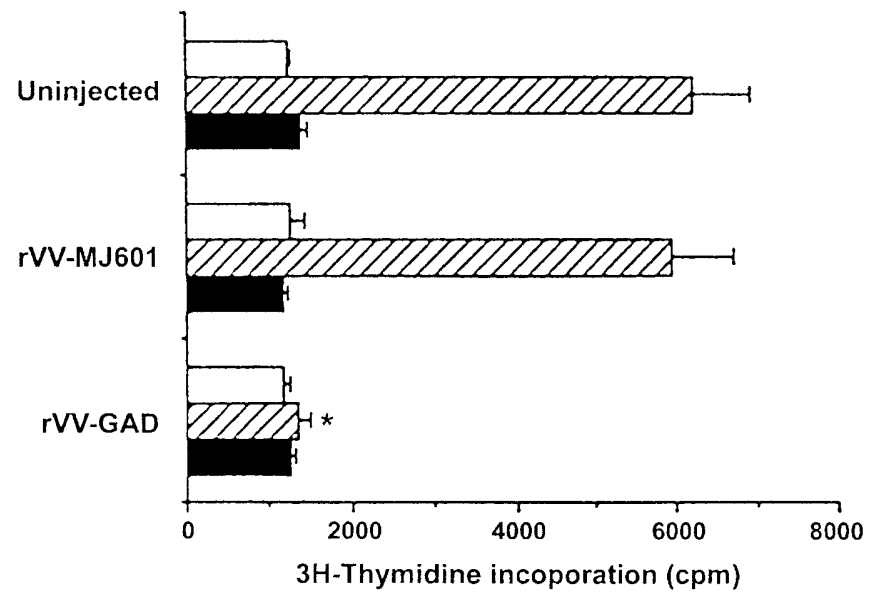

Fig. 5. The T-cell immune response to GAD was diminished in rVV-GAD65-treated NOD mice. Splenocytes isolated from NOD mice 8 weeks after injection of rVV-GAD65 or rVVMJ601 were reacted with GAD protein (GAD, cross-hatched columns), ovalbumin (OVA $\square$ ) or control media (media $\Rightarrow$ ). The cells were pulsed with $1 \mu \mathrm{Ci}$ of $\left[{ }^{3} \mathrm{H}\right]$-thymidine. Proliferation was determined by $\left[{ }^{3} \mathrm{H}\right]$-thymidine uptake. Results are shown as cpm $\pm \mathrm{SD}$ of three experiments using two mice for each group. The proliferative response of splenocytes from uninjected mice were examined as a control. ${ }^{*} p<0.01$ as compared to rVV-MJ601-injected mice

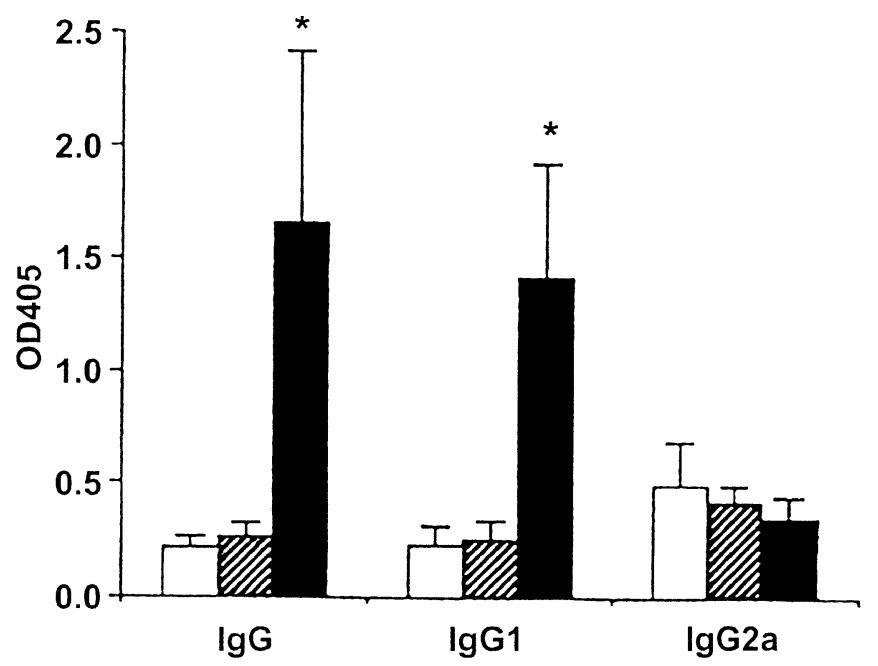

Fig. 6. Treatment of NOD mice with rVV-GAD65 increased IgG1 anti-GAD antibodies. Sera from rVV-GAD65- $\Leftrightarrow)$ and rVV-MJ601-vaccinated (cross-hatched columns) NOD mice and uninjected $(\square)$ NOD mice were analysed for anti-GAD $\mathrm{IgG}, \operatorname{IgG} 1$ and $\mathrm{IgG} 2 \mathrm{a}$ antibodies by ELISA as described in the Methods. Values are shown as means \pm SD of 7 mice. $* p<0.001$ as compared to rVV-MJ601-injected mice

crease in the amount of $\operatorname{IgG}$ antibodies to GAD in the serum of rVV-GAD65-injected mice as compared to $\mathrm{rVV}$-MJ601-injected and uninjected mice. When the isotypes of the IgG antibodies to GAD were examined, the amount of the IgG1 subtype was specifically increased in rVV-GAD65-immunized NOD mice, whereas the $\operatorname{IgG} 2 \mathrm{a}$ subtype did not change compared to rVV-MJ601-injected NOD mice
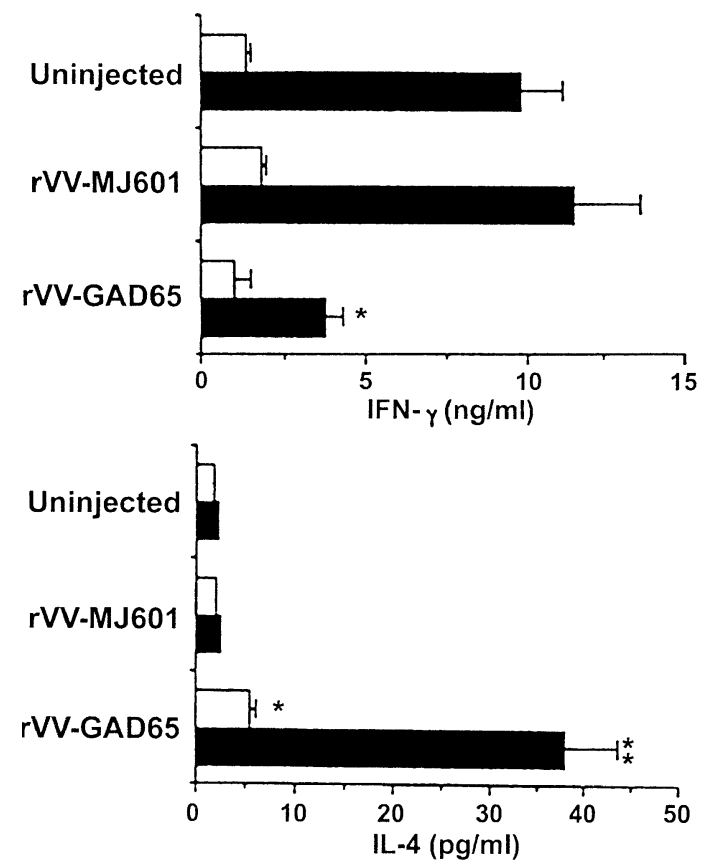

Fig. 7. Treatment of NOD mice with rVV-GAD65 increased IL-4 production and decreased IFN- $\gamma$ production. Splenocytes were isolated from five mice from each group 8 weeks after vaccination and stimulated with $(+\mathrm{GAD} \Rightarrow)$ or without $(-\mathrm{GAD} \square)$ recombinant GAD65 protein for 3 days. The production of IFN- $\gamma$ and IL- 4 was determined in the culture supernatant by ELISA. Values are shown as mean \pm SD of 5 mice. ${ }^{*} p<0.05$, $* * p<0.001$ as compared to rVV-MJ601-injected mice

(Fig. 6). These observations suggest that the rVVGAD65 immunisation enhanced the Th2 immune response. This possibility was supported when we analysed IFN- $\gamma$ and IL-4 release in vitro following GAD stimulation of splenocytes collected from NOD mice 8 weeks after rVV-GAD65- or rVV-MJ601 injection. The production of IL-4 increased, whereas the production of IFN- $\gamma$ decreased in the splenocytes of rVV-GAD65-injected mice compared to splenocytes from rVV-MJ601-injected NOD mice (Fig. 7). These results suggest that immunisation of NOD mice with rVV-GAD65 induces a Th2 immune response in an antigen-specific manner.

rVV-GAD65 immunisation of NOD mice induces regulatory cells. Finally, we examined whether immunisation of NOD mice with rVV-GAD65 might promote the generation of regulatory cells, resulting in the protection of beta cells from destruction by effector $\mathrm{T}$ cells. We isolated splenocytes from rVV-GAD65- or rVV-MJ601-injected mice at 20 weeks of age and transfused these splenic lymphocytes alone, or together with an equal number of splenocytes from acutely diabetic NOD mice, into 6- to 8-week-old NOD.scid mice. We found that $13 \%(1 / 8)$ of the NOD.scid recipients of splenic lymphocytes from rVV-GAD65-treated NOD mice developed diabetes, whereas $86 \%(6 / 7)$ of NOD.scid recipients of splenic lymphocytes from 
Table 1. Splenocytes from rVV-GAD65-treated NOD mice prevented the adoptive transfer of diabetes

\begin{tabular}{lllllllcc}
\hline Donor splenocytes & \multicolumn{3}{l}{ Incidence of diabetes - weeks after transfer } & & \\
\cline { 2 - 8 } & 1 & 2 & 3 & 4 & 5 & 6 & 7 & $8(\%)$ \\
\hline rVV-MJ601 & $0 / 7$ & $0 / 7$ & $1 / 7$ & $3 / 7$ & $5 / 7$ & $6 / 7$ & $6 / 7$ & $6 / 7(86)$ \\
rVV-GAD65 & $0 / 8$ & $0 / 8$ & $0 / 8$ & $1 / 8$ & $1 / 8$ & $1 / 8$ & $1 / 8$ & $1 / 8(13)$ \\
Diabetic & $0 / 7$ & $0 / 7$ & $1 / 7$ & $1 / 7$ & $3 / 7$ & $6 / 7$ & $7 / 7$ & $7 / 7(100)$ \\
rVV-MJ601 + Diabetic & $0 / 11$ & $0 / 11$ & $1 / 11$ & $3 / 11$ & $6 / 11$ & $10 / 11$ & $10 / 11$ & $10 / 11(91)$ \\
rVV-GAD65 + Diabetic & $0 / 9$ & $0 / 9$ & $0 / 9$ & $1 / 9$ & $2 / 9$ & $2 / 9$ & $3 / 9$ & $3 / 9(33)$ \\
\hline
\end{tabular}

Splenocytes $\left(1 \times 10^{7}\right.$ cells $)$ isolated from rVV-GAD65-treated or rVV-MJ601-treated NOD mice at 20 weeks of age were transferred alone or together with splenocytes $\left(1 \times 10^{7}\right.$ cells $)$ from acutely diabetic NOD mice into NOD.scid mice. The development of diabetes in the recipients was monitored
rVV-MJ601-treated NOD mice developed diabetes (Table 1). In addition, 33\% (3/9) of the NOD.scid recipients of splenic lymphocytes that included cells from rVV-GAD65-injected NOD mice developed diabetes, whereas $91 \%(10 / 11)$ of the NOD.scid recipients of splenic lymphocytes that included cells from rVV-MJ601-injected mice developed diabetes (Table 1). These results imply that the vaccine might alter disease expression in NOD mice by altering cellular immune function, such as by inducing a regulatory $\mathrm{T}$ cell population.

\section{Discussion}

Tolerance induction of autoreactive $\mathrm{T}$ cells against specific target autoantigens has been attempted as a method to prevent autoimmune disease. In Type I diabetes, GAD and insulin have been identified as major autoantigens and tolerisation against these autoantigens has been attempted as a method for the prevention of the disease $[14,32]$. It has been reported that administration of purified GAD protein or peptide or insulin protein or peptide to NOD mice by a variety of routes can tolerise the $\mathrm{T}$ cell-mediated immune response against pancreatic beta cells, resulting in the prevention or delay of the development of insulitis and diabetes [28, 29, 33, 34, 35, 36, 37, 38, 39]. In many cases, the preventive effect was found to be associated with a Th2 shift $[14,32]$.

An extension of this idea is the vaccination of NOD mice with plasmid DNA encoding GAD or insulin although such studies have inconsistent results. Some showed that intramuscular injection of a plasmid encoding GAD65 resulted in the prevention of diabetes $[40,41]$; others showed that injection of a plasmid encoding GAD65 was not effective [42, 43, 44]. However, a plasmid encoding GAD65 was effective only if the plasmid also contained DNA encoding IL-4 [42, 44]. Some studies showed that an insulin gene vaccine had a preventive effect $[43,45]$; another showed that an insulin gene vaccine accelerated the onset of diabetes [42]. These different results might be due to different amounts of autoantigen gene expression in the vaccinated mice, as the transfection efficiency by intramuscular injection is quite low. Therefore, in our study, we used vaccinia virus as a carrier for the GAD65 autoantigen gene for the vaccination of NOD mice because the vaccinia virus vector has a high transduction efficiency and induces a strong immune response. We found that a single immunisation of three-week-old NOD mice with rVV-GAD65 resulted in the almost complete prevention of autoimmune diabetes.

Our first question was whether vaccinia virus itself might cause a non-specific immune disturbance that might result in the prevention of autoimmune diabetes in NOD mice immunised with rVV-GAD65. To exclude this possibility, we immunised NOD mice with recombinant vaccinia virus containing the vector pMJ601 without the GAD65 cDNA insert. A total of $85 \%$ of these mice became diabetic, an incidence similar to that in untreated NOD mice, although the onset of disease was delayed. Similarly, $80 \%$ of mice that received another control treatment, rVV expressing the unrelated protein NS1 of the Kilham rat virus, developed diabetes. This result indicates that the prevention of autoimmune diabetes by rVV-GAD65 immunisation is not due to a non-specific immune disturbance but an antigen-specific effect.

Our second question was whether the prevention of autoimmune diabetes by immunisation with GADexpressing vaccinia virus is dose-dependent or agedependent. To answer the question of age, we immunised female NOD mice with rVV-GAD65 before ( 3 weeks of age) or after (8-9 weeks of age) the development of insulitis. We found that immunisation with rVV-GAD65 before the development of insulitis almost completely prevented diabetes, whereas immunisation after the development of insulitis did not prevent diabetes indicating that immunisation with this vaccine at an early age, before insulitis, is required for the preventive effect to occur. The lack of preventive effect by rVV-GAD65 in 8-9 week-old NOD mice might be due to the pre-existing increase in the Th1 immune response at this age and the failure to sufficiently induce a Th2 immune response in these 
animals. To determine whether the dose of rVVGAD65 is critical for the prevention of diabetes in NOD mice, we immunised three-week-old NOD female mice with 3 different doses of rVV-GAD65. We found that only $7 \%$ of mice immunised with a dose of $5 \times 10^{7}$ PFU of rVV-GAD65 developed diabetes, while lower dosages $\left(2.5 \times 10^{7} \mathrm{PFU}\right.$ and $\left.1 \times 10^{7} \mathrm{PFU}\right)$ resulted in a reduced effect $(53 \%$ and $67 \%$ diabetic, respectively). This result indicates that the dose of GAD-expressing vaccinia virus is critical for the prevention of autoimmune diabetes.

Our third question was what mechanisms are involved in the prevention of autoimmune diabetes in NOD mice by rVV-GAD65 treatment. We examined the proliferative response of splenocytes from NOD mice infected with rVV-GAD65 to GAD protein. We found that these splenocytes did not respond to GAD, implying that GAD-reactive $\mathrm{T}$ cells were tolerised in the rVV-GAD65-treated mice. We then examined the humoral immune response against GAD in the sera of NOD mice infected with rVV-GAD65. We found that the amount of the IgG1 subtype was increased in the rVV-GAD65-infected mice $(p<0.001)$, whereas the IgG2a subtype was not changed, suggesting that immunisation of NOD mice with rVV-GAD65 results in the enhancement of the Th2 immune response. To determine whether the Th2 immune response is truly involved in the disease process, we examined IFN- $\gamma$ and IL-4 release in vitro following GAD stimulation of splenocytes obtained from NOD mice infected with rVV-GAD65. We found that the production of IL-4 increased, whereas the production of IFN- $\gamma$ decreased, indicating that the immunisation of NOD mice with rVVGAD65 induced a Th2 immune response in an antigenspecific manner. Our finding is consistent with the results of previous reports on NOD mice treated with GAD protein systemically, nasally or orally $[28,29,33$, $34,35]$ or vaccinated with GAD encoding plasmid DNA [41]. Th2 immune responses induced by rVVGAD immunisation could play an important role in the prevention of autoimmune diabetes in this animal model. In addition, the increase in anti-GAD antibodies could synergise the preventive effect, as the administration of anti-GAD antibody has been shown to prevent the development of diabetes in NOD mice [46].

We then examined whether rVV-GAD65 treatment induced the generation of regulatory cells that could suppress diabetogenic effector $\mathrm{T}$ cells. We adoptively co-transferred splenocytes from rVV-GAD65 immunised NOD mice with splenocytes from acutely diabetic NOD mice into NOD.scid mice. The splenocytes from the rVV-GAD65 immunised mice were found to inhibit the transfer of diabetes by splenocytes from acutely diabetic NOD mice. This result suggests that immunisation with rVV-GAD65 could generate a regulatory population. GAD expressed in rVV-GAD65infected cells could present GAD peptide in conjunction with $\mathrm{MHC}$ antigens and stimulate the regulatory cells. However, the precise mechanism involved in the generation of regulatory $\mathrm{T}$ cells in rVV-GAD65-treated mice remains to be determined.

In conclusion, we have developed a recombinant vaccinia virus expressing GAD65. Immunogene therapy using this rVV-GAD65 resulted in the almost complete prevention of autoimmune diabetes in NOD mice by a single injection. Further studies on the mechanisms for this preventive effect suggest that the therapeutic effect of rVV-GAD65 was due to the induction of immunological tolerance through the active suppression of effector T cells by the induction of Th2 immune responses, rather than clonal deletion or clonal anergy. As no serious side-effects were found in healthy human subjects that received percutaneous administration of vaccinia virus $[23,24]$, a recombinant vaccinia virus expressing GAD might have therapeutic value for the prevention of Type I diabetes.

Acknowledgements. We gratefully acknowledge B. Moss for providing the pMJ601 vector, D. Kaufman for providing the mouse GAD65 cDNA, K. Hirasawa for performing the immunocytochemistry, and A. L. Kyle for editorial assistance. This work was supported by grants from the Canadian Institutes of Health Research (MA 9584), the Juvenile Diabetes Foundation International, the Korean Green Cross Corporation, the National Institutes of Health (DK 45735 and DK 53015-01) and the Alberta Heritage Foundation for Medical Research. J. W. Yoon holds a Canada Research Chair in Diabetes.

\section{References}

1. Schranz DB, Lernmark A (1998) Immunology in diabetes: an update. Diabetes Metab Rev 14: 3-29

2. Tisch R, McDevitt H (1993) Insulin-dependent diabetes mellitus. Cell 85: 291-297

3. Rossini AA, Greiner DL, Friedman HP, Mordes JP (1993) Immunopathogenesis of diabetes mellitus. Diabetes Rev 1: 43-75

4. Castano L, Eisenbarth GS (1990) Type 1 diabetes: A chronic autoimmune disease of human, mouse and rat. Annu Rev Immunol 8: 647-679

5. Yoon JW, Jun HS (1998) Insulin-dependent diabetes mellitus: experimental animal models. In: Roitt IM, Delves PJ (eds) Encyclopedia of immunology. 2nd edn. Academic Press, London, pp. 1390-1398

6. Bach JF (1994) Insulin-dependent diabetes mellitus as an autoimmune disease. Endocr Rev 15: 516-542

7. Leiter EH, Serreze DV, Prochaz KAM (1990) The epidemiology of diabetes in NOD mice. Immunol Today 11: 147149

8. Delovitch TL, Singh B (1997) The nonobese diabetic mouse as a model of autoimmune diabetes: immune dysregulation gets the NOD. Immunity 7: 727-738

9. Wong FS, Janeway CA (1999) Insulin-dependent diabetes mellitus and its animal models. Curr Opin Immunol 11: 643-647

10. Haskins K, McDuffie M (1990) Acceleration of diabetes in young NOD mice with a $\mathrm{CD}^{+}{ }^{+}$islet-specific $\mathrm{T}$ cell clone. Science 249: 1433-1436

11. Nagata M, Santamaria P, Kawamura T, Utsugi T, Yoon JW (1994) Evidence for the role of $\mathrm{CD}^{+}$cytotoxic T cells in 
the destruction of pancreatic beta cells in NOD mice. J Immunol 152: 2942-2050

12. Nagata M, Yoon JW (1992) Studies on autoimmunity for T cell-mediated $\beta$ cell destruction: Distinct differences in $\beta$ cell destruction between $\mathrm{CD}^{+}{ }^{+}$and $\mathrm{CD}^{+}{ }^{+} \mathrm{T}$ cell clones derived from lymphocytes infiltrating the islets of NOD mice. Diabetes 41: 998-1008

13. Atkinson MA, Maclaren NK (1993) Islet cell autoantigens in insulin-dependent diabetes. J Clin Invest 92: 240-248

14. Bach J-F, Chatenoud L (2001) Tolerance to islet autoantigens in type 1 diabetes. Annu Rev Immunol 19: 131161

15. Fenner F (1989) Risks and benefits of vaccinia virus use in the worldwide smallpox eradication campaign. Res Virol 140: 465-466

16. Henderson DA, Arita I (1985) Utilization of vaccine in the global eradication of smallpox. In: Quinnan GV Jr (ed) Vaccinia viruses as vectors for vaccine antigens. Elsevier, New York, pp. 61-67

17. Moss B (1996) Genetically engineered poxviruses for recombinant gene expression, vaccination, and safety. Proc Natl Acad Sci USA 93: 11341-11348

18. Paoletti E (1996) Applications of pox virus vectors to vaccination: An update. Proc Nat Acad Sci USA 93: 1134911353

19. Brown F, Schild GC, Ada GL (1986) Recombinant vaccinia viruses as vaccines. Nature 319: 549-550

20. Moss B (1991) Vaccinia virus: a tool for research and vaccine development. Science 252: 1662-1667

21. Hany M, Oehen S, Schulz M et al. (1989) Anti-viral protection and prevention of lymphocytic choriomeningitis or of the local footpad swelling reaction in mice by immunization with vaccinia recombinant virus expressing LCMV-WE nucleoprotein or glycoprotein. Eur J Immunol 19: 417-424

22. Kulkarni AB, Collins PL, Bacik I et al. (1995) Cytotoxic T cells specific for a single peptide on the M2 protein of respiratory syncytial virus are the sole mediators of resistance induced by immunization with M2 encoded by a recombinant vaccinia virus. J Virol 69: 1261-1264

23. Cooney EL, McElrath MJ, Corey L et al. (1993) Enhanced immunity to human immunodeficiency virus (HIV) envelope elicited by a combined vaccine regimen consisting of priming with a vaccine recombinant expressing HIV envelope and boosting with gp/60 protein. Proc Natl Acad Sci USA 90: 1882-1886

24. Cooney EL, Collier AC, Greenberg PD et al. (1991) Safety of and immunological response to a recombinant vaccinia virus vaccine expressing HIV envelope glycoprotein. Lancet 337: 569-572

25. Lee DS, Tian J, Phan T, Kaufman DL (1993) Cloning and sequence analysis of a murine cDNA encoding glutamic acid decarboxylase (GAD65). Biochim Biophys Acta 1216: 157-160

26. Earl PL, Moss B (1991) Generation of recombinant vaccinia viruses. In: Ausubel FM (ed) Current protocols in molecular biology. Greene Publishing Associates, Wiley Interscience, New York, pp. 16.17.1-16.17.16

27. Chung YH, Jun HS, Son M et al. (2000) Cellular and molecular mechanism for Kilham rat virus-induced autoimmune diabetes in DR-BB rats. J Immunol 165: 2866-2876

28. Tisch R, Yang XD, Singer SM, Liblau RS, Fugger L, McDevitt HO (1993) Immune response to glutamic acid decarboxylase correlates with insulitis in non-obese diabetic mice. Nature 366: $72-75$
29. Elliott JF, Qin HY, Bhatti S et al. (1994) Immunization with the larger isoform of mouse glutamic acid decarboxylase (GAD67) prevents autoimmune diabetes in NOD mice. Diabetes 43: 1494-1499

30. Jun HS, Yoon CS, Zbytnuik L, van Rooijen N, Yoon JW (1999) Role of macrophages in T cell-mediated autoimmune diabetes in nonobese diabetic mice. J Exp Med 189: 347-358

31. Yoon JW, Rodrigues MM, Currier C, Notkins A (1982) Long-term complications of virus-induced diabetes mellitus in mice. Nature 296: 566-569

32. Cooke A, Phillips JM, Parish NM (2001) Tolerogenic strategies to halt or prevent type 1 diabetes. Nat Immunol 2: $810-815$

33. Kaufman DL, Clare-Salzler M, Tian J et al. (1993) Spontaneous loss of T-cell tolerance to glutamic acid decarboxylase in murine insulin-dependent diabetes. Nature 366: 69-72

34. Ma SW, Zhao DL, Yin ZQ et al. (1997) Transgenic plants expressing autoantigens fed to mice to induce oral immune tolerance. Nat Med 3: 793-796

35. Tian J, Atkinson MA, Clare-Salzler M et al. (1996) Nasal administration of glutamate decarboxylase (GAD65) peptides induces Th2 responses and prevents murine insulindependent diabetes. J Exp Med 183: 1561-1567

36. Tian J, Clare-Salzer M, Herschenfeld A et al. (1996) Modulating autoimmune responses to GAD inhibits disease progression and prolongs graft survival in diabetes-prone mice. Nat Med 2: 1348-1353

37. Daniel D, Wegmann DR (1996) Protection of nonobese diabetic mice from diabetes by intranasal or subcutaneous administration of insulin peptide B-(9-23). Proc Natl Acad Sci USA 93: 956-960

38. Zhang ZJ, Davidson L, Eisenbarth G, Weiner HL (1991) Suppression of diabetes in nonobese diabetic mice by oral administration of porcine insulin. Proc Natl Acad Sci USA 88: $10525-10526$

39. Harrison LC, Dempsey-Collier M, Kramer DR, Takahashi K (1996) Aerosol insulin induces regulatory CD8 gamma delta $\mathrm{T}$ cells that prevent murine insulin-dependent diabetes. J Exp Med 184: 2167-2174

40. Balasa B, Boehm BO, Fortnagel A et al. (2001) Vaccination with glutamic acid decarboxylase plasmid DNA protects mice from spontaneous autoimmune diabetes and B7/CD28 costimulation circumvents that protection. Clin Immunol 99: 241-252

41. Filippova M, Liu J, Escher A (2001) Effect of plasmid DNA injection on cyclophosphamide-accelerated diabetes in NOD mice. DNA Cell Biol 20: 175-181

42. Weaver DJ Jr, Liu B, Tisch R (2001) Plasmid DNAs encoding insulin and glutamic acid decarboxylase 65 have distinct effects on the progression of autoimmune diabetes in nonobese diabetic mice. J Immunol 167: 586-592

43. Bot A, Smith D, Bot S et al. (2001) Plasmid vaccination with insulin B chain prevents autoimmune diabetes in nonobese diabetic mice. J Immunol 167: 2950-2955

44. Tisch R, Wang B, Weaver DJ et al. (2001) Antigen-specific mediated suppression of $\beta$ cell autoimmunity by plasmid DNA vaccination. J Immunol 166: 2122-2132

45. Urbanek-Ruiz I, Ruiz PJ, Paragas V et al. (2001) Immunization with DNA encoding an immunodominant peptide of insulin prevents diabetes in NOD mice. Clin Immunol 100: 164-171

46. Menard V, Jacobs H, Jun HS, Yoon JW, Kim SW (1999) Anti-GAD monoclonal antibody delays the onset of diabetes mellitus in NOD mice. Pharm Res 16: 1059-1066 